\title{
Photoemission from Metal Nanoparticles
}

\author{
Igor Protsenko and Alexander Uskov \\ Lebedev Physical Institute, Plasmonics ltd. \\ Russia
}

\section{Introduction}

It is well-known that oscillations of electron density of metal nanoparticles have resonant frequency in the visible or in the near IR spectral region: this is the localized plasmon resonance (LPR). New and rapidly developed branch of modern physics called "Nanoplasmonics", is dedicated to investigations of optical and electro-physical phenomena related with the excitation of LPR and others similar resonances in metal nanoparticles and nanostructures. Appearance of LPR is caused by the charge on the surface of nanoparticles (Brongersma \& Kik, 2007; Klimov, 2009; Maier, 2007; Novotny \& Hecht, 2006): the surface of the nanoparticle builds "potential well" for oscillations of the electron density of the metal. For small particles (which typical size is less than or about $40 \mathrm{~nm}$ ) the frequency of LPR depends weakly on the size of nanoparticles and strongly on their shape, metal and external environment. LPR can be excited by the external electromagnetic field (EMF). Energy of LPR is stored in oscillations of the electron density of nanoparticles and in the EMF induced by oscillations of the electron density. When LPR is excited, the density of energy of EMF inside the nanoparticle and near it, on the distance of the order or less than the wavelength of EMF, is approximately $Q$ times greater (Wang, 2006) than the energy density of the external FMF. Here $\mathrm{Q}$ is the quality factor of LPR, Q depends on losses due to absorption of EMF by the metal of nanoparticle and on the radiation of EMF from the nanoparticle to the environment. Usually $\mathrm{Q} \leq 10$ in experiments (Brongersma \& Kik, 2007; Hövel et al., 1993; Klimov, 2009; Maier, 2007; Novotny \& Hecht, 2006; Schuller et al, 2010), though some theoretical estimations predict the maximum value of $Q$ about several tens (Khlebtsov, 2008). With the excitation of LPR nanoparticle behaves as a "nano-cavity" for EMF. However in a difference from the usual cavity, as Fabri-Perot cavity, a near-field presents in the "nano-cavity". Near field is bounded with charges, near field zone is located on the distance $\leq \lambda$, where $\lambda$ is the wavelength of EMF. Resonant (or „plasmonic“ (Brongersma \& Kik, 2007; Khlebtsov, 2008; Klimov, 2009; Maier, 2007; Novotny \& Hecht, 2006)) properties of metal nanoparticles and the concentration of EMF near and inside particles are reasons for prediction and experimental observation a number of new phenomena (Brongersma \& Kik, 2007; Khlebtsov, 2008; Klimov, 2009; Kneipp et al., 2006; Maier, 2007; Novotny \& Hecht, 2006) as, for example, giant Relay scattering (Kneipp et al., 2006). New optoelectronic devices with plasmonic nanoparticles were designed and created, as sensors (Brongersma \& Kik, 2007; Homola, 1999; Klimov, 2009; Kneipp et al., 2006; Maier, 2007; Novotny \& Hecht, 2006), nano-scaled lasers (Bergman \& Stockman, 2003; Noginov et al. 2009; Oulton et al., 2009; Protsenko et al., 2005), high efficient solar cells (Atwater \& Polman, 2009; Catchpole \& Polman, 2008; Pors, 2011). 
Nanoparticles in optical devices can be considered as „nano-antennas“ (Brongersma \& Kik, 2007; Greffet, 2005; Klimov, 2009; Maier, 2007; Mühlschlegel et al., 2005; Novotny \& Hecht, 2006). It is important and interesting to investigate also electrophysical properties of contacts of plasmonic nanoparticles with environment (for example, a surface) surrounding (supporting) nanoparticles. Transport of carriers (electrons or holes) or transport of the energy from the nanoparticle to the environment and back may cause noticerable influence to properties of the device with nanoparticles. Such influence may be positive or negative up to complete destruction of LPR and related phenomena. For example the effect of increase of photo-luminescence due to addition of metal nano-particles in luminescing media (as solutions of dye moleculas) is well-known. However if the distance between the nanoparticle and the luminescing obgect is small (less than few $\mathrm{nm}$ ) the luminescence can be fully dumped due to non-radiated recombination (Brongersma \& Kik, 2007; Klimov, 2003, 2009; Maier, 2007; Novotny \& Hecht, 2006).

Various constructions of "plasmonic" solar cells have been suggested. Both optical and electro-physical properties of metal nanoparticles are used in such solar cells, for example, for commutation (electrical connection) of cascades of the solar cell through metal nanoparticles using also "plasmonic" concentration of the light arround nanoparticles (Rand et al., 2006). Solar cell efficiency can be increased due to injection of carriers, photo-induced in metal nanoparticles, to the semiconductor substrate, while LPR is excited in nanoparticles (Westphalen et al., 2006). However the transport of carriers through the contacts of metal nanoparticles with the substrate are less studied than optical properties of metal nanoparticles. It is related with complexity of problems arisen in theoretical and experimental studies of electro-physical properties of nano-scaled objects. Investigation of the carrier transport and other electrophysical properties of junctions between substrates and metal nanoparticles must be carried out together with investigation of optical properties of nanoparticles. This is necessary for modeling plasmonic optoelectronic devices as solar cells (Monestier et al., 2007), photodetectors, nano-scaled LEDs and nano-lasers. This work gives an example of such "joint" study of optical and electro-physical phenomena in plasmonics.

An example of phenomena appeared at the interface between the nanoparticle and the environment is a photoemission from the nanoparticle studied here theoretically. The photoemission from the nanoparticle may be quite different from the photoemission from "large" (respectively to the wavelength of EMF) metal structures (as, for example, continues metal films widely used in photodetectors). One can note three major differences. First, the EMF inside the nanoparticle and near it is enhanced at the excitation of LPR. Second, the ratio of the area of surface of the nanoparticle to the volume of the nanoparticle is greater than for large "bulk" metal structures. This is important because of the "surface" photoeffect is more important for the photoemission than the "bulk" photo-effect (Brodsky, 1973). The "surface" photo-effect is occurred when the photon is absorbed at the collision of the electron with the surface, while the "bulk" photo-effect takes place when the photon is absorbed inside the metal at the collision of the electron with the lattice (i.e. with the phonon) or with another electron. Usually the "bulk" photo-effect is not taken into consideration at calculations of photo-emission as, for example, in the case of photoemission from metal films (Brodsky, 1973). Third, in order to provide the surface photoemission the electric field must be non-parallel (perpendicular, at best) to the surface 
of the metal. This condition can be easily satisfied for nanoparticles than for continues metal films. Thus for metal nano-particles one can expect to obtain larger number of photoelectrons per unit of mass that it is obtained for bulk metal structures as metal films in wellknown photo-detectors (She, 1981; Soole \& Schumacher, 1991). Increase of the efficiency of photo-detectors by metal nano-particles helps, in particular, to increase the sensitivity of photo-detectors in the near and far IR spectral regions, which is important practical problem (Piotrowski et al., 1990; Yu et al., 2006).

For the study of photoemission from nano-particles and, in particular, for determination of conditions of the maximum of photoemission one has to know the cross-section of the photoemission from the nanoparticle, which is the main subject of calculations presented below. We present results for the case of the surface photo-emission from metal nanoparticle at the excitation of LPR and show, as an example, that the photo-emission from metal nano-particles into $\mathrm{p}$-doped $\mathrm{Si}$ is much more efficient that the photo-emission from the bulk metal film. It is worth to note that micro- and nano-structures on surfaces of metal photo-detectors is widely used for enhancement of the photo-emission, in particular, in photo-cathodes based on $\mathrm{A}^{\mathrm{III}} \mathrm{BV}$ semiconductors appeared at the beginning of 70-th years. It was shown in several publications (see, for example, the bibliography in (Schelev, 2000)) that the photo-emitting metal film of such photo-cathodes has dispersed structure. It is also well-known that all such photo-cathodes have larger photo-current respectively to photocathodes with photo-emission from flat metal layers. However LPR can be hardly excited in "dispersed" metal structures on surfaces of such photo-cathodes. These structures have good electric contact with surfaces, but LPR can be excited in metal nanoparticles electrically isolated from the environment. Thus the increase of the photo-emission due to the "concentration" of EMF at nano-structured surfaces of well-known photo-cathodes is hardly possible. Larger photoemission current of well-known photo-cathodes with dispersed surfaces was obtained due to lager surface, available for the photoemission. If LPR can be excited in nano-structures at surfaces of photo-cathodes, it will lead to more increase in the photoemission; this is why it is interesting and important to study photo-cathodes with LPR excited in nanoparticles. It was already suggested to use plasmonic properties of nanoparticles for increase of the efficiency of photo-detectors (Hetterich, 2007), however without consideration of photoemission from nano-particles. Increase of the efficiency of photoemission to vacuum from nano-particles has been observed experimentally (Nolle, 2004, 2005), it was suggested to use this effect for increase the efficiency of photo-detectors.

Here we carry out the analysis following by the well-known theory of photoemission (Brodsky, 1973). In particular we will find general expression for the probability amplitude and calculate the cross-section of the photoemission from metal nano-particle. Calculation shows the two order of magnitude increase of the photoemission current from gold nanoparticles to p-doped Si in comparison with the photocurrent from continues film of Au. We generalize the result of (Brodsky, 1973) for the probability of photoemission by taking into account changes (jumps) of EMF and electron mass at the surface of the nanoparticle. Taking into consideration these surface phenomena we see that the cross-section of photoemission is changed (increased) several times with the respect to the case, when these "jumps" on the surface are neglected. Careful consideration of surface phenomena is important particularly for the photoemission from nanoparticles because of their large surface to volume ratio. 
The probability and the cross-section of the photoemission found here let us to calculate the photocurrent from the layer of metal nanoparticles. We use analytical approach following details of physics of the increase of the photoemission from nanoparticles at LPR. It is shown, for example, that the increase of the cross-section of the photoemission is related not only with "concentration" of EMF but also with changes of EMF and free electron mass on the surface of nano-particle. It turns to be that quantum-mechanical interference phenomena in the dynamics of electron passing through the interface became important at the photoemission. Analytical approach lets us to separate three contributions in the photoemission: (1) the increase of the probability of photoemission, (2) the increase of EMF due to the excitation of LPR and (3) the factor of the shape of the surface of the nanoparticle. In principle, one can control photo-emission by changing these three factors. For example, by changing the shape of the nanoparticle one can shift the maximum of the photocurrent spectrum. However detailed analysis of possibilities of controlling the photoemission is out of the scope of this paper. Analytical approach lets us to take into consideration many peculiarities of optical "plasmonic" phenomena, for example, dynamical depolarization and radiation dumping phenomena. Analytical results can be used as test models for verification of numerical results found for more complex cases of photoemission as, for example, nondipole approach at the interaction of nanoparticles with EMF.

Here we discuss the photoemission to vacuum or to the homogeneous medium surrounding nanoparticle. However the same approach may be applied for the analysis of other more complicated cases of the carrier transport at presence of nanoparticles. For example the nanoparticle may catch the carrier from the environment; the photoemission may occur as the tunnelling through the potential barrier (Nolle, 2007) also when the nano-particle stays near the surface of the semiconductor and separated from it by the tunnel layer. The last structure is typical for problems related with the increase of the efficiency of solar cells by meal nanoparticles (Atwater \& Polman, 2009; Catchpole \& Polman, 2008; Pors, 2011).

General expression for the probability amplitude of the photoemission with taking into account changes in the EMF and the mass of electron at the nanoparticle-environment interface is derived in the next subsection. Explicit expression for the probability amplitude is found for the step potential at the metal-environment interface. This result is used for the calculation of the cross-section of photoemission in the following sub-section. Example of the photoemission from spherical gold nano-particles into p-doped Si is considered after that. Results are summarised and directions for future studies of the photoemission from nano-particles are discussed in conclusion.

In order to calculate the probability of the photoemission from the metal we, following (Brodsky, 1973), use standard quantum-mechanic perturbation theory (Landau \& Lifshitz, 1997) where the perturbation is the interaction of the electron with classical electromagnetic field at the metal-environment interface. We neglect by the curvature of the surface of the metal with the respect to de Broglie wavelength of the electron, i.e. we consider metalenvironment interface as a flat surface. The key point is the calculation of "unperturbed" wave functions of the electron in order to insert them into the ready expression for the probability amplitude found from the perturbation theory. We found wave functions analytically supposing a step potential barrier between the metal and the environment. This lets us to obtain final expression for the cross-section of the photoemission "in quadratures" i.e. as an explicit expression containing the integral. The electromagnetic field causing the 
photoemission appears as a multiplier in this expression. Then we calculate the electromagnetic field in the nanoparticle and in the vicinity of it using the approach of classical electrodynamics well-known in plasmonics (Klimov, 2009).

\section{Probability amplitude of the photoemission accounting "jumps" on the interface}

\subsection{General expression for probability amplitude of photoemission}

Expression for the probability amplitude $\mathrm{C}_{+}(\infty)$ of the photoemission of the electron was found in (Brodsky, 1973) by the perturbation theory. Electron moves in the medium (in the metal) along axes $z$ perpendicular to the interface with the external environment. The electron interacts with the EMF of frequency $\omega$,

$$
\mathrm{C}_{+}(\infty)=\frac{|\mathrm{e}| \mathrm{m}}{\hbar \omega \mathrm{W}_{1}} \int_{-\infty}^{\infty} \mathrm{dz}\left(\mathrm{E}_{\mathrm{m}} \frac{\mathrm{d} \Psi_{0}}{\mathrm{dz}} \Psi_{1-}+\frac{1}{2} \Psi_{1-} \Psi_{0} \frac{\mathrm{dE}_{\mathrm{m}}}{\mathrm{dz}}\right)
$$

Here e is the (negative) charge of the electron, $\mathrm{m}$ is the mass of the electron. The interface of the medium with the environment is described by the one dimentional potential barrier $\mathrm{V}(\mathrm{z})$, see Fig.1. Effective mass of the electron is changed on the interface, so that $\mathrm{m}=\mathrm{m}(\mathrm{z})$;

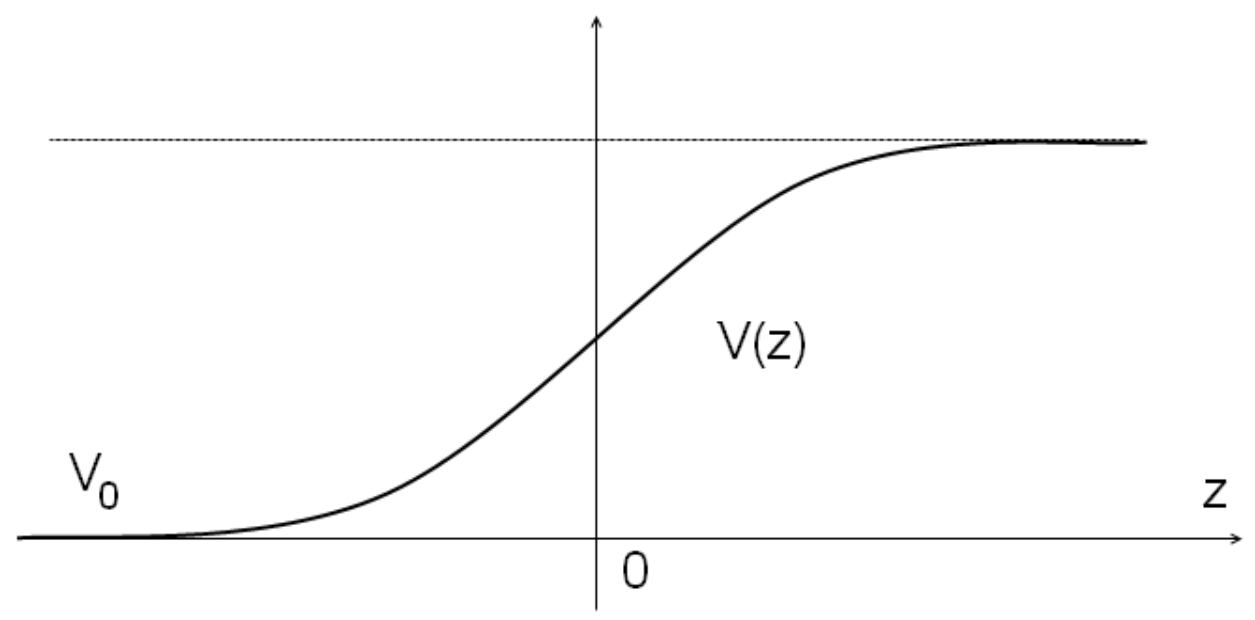

Fig. 1. Potential barrier where the electron moves.

$\Psi_{0}, \Psi_{1 \pm}$ are wave functions of the electron in states with the energy $E_{i}, i=0,1$ below and above the barrier respectively, $\mathrm{E}_{\mathrm{m}} \equiv \mathrm{E} / \mathrm{m}(\mathrm{z}), \mathrm{E}$ is the amplitude of the component of the electric field polarized along axes $\mathrm{z}$,

$$
\mathrm{W}\left(\Psi_{1-} \Psi_{1+}\right)=\Psi_{1-} \frac{\mathrm{d} \Psi_{1+}}{\mathrm{dz}}-\Psi_{1+} \frac{\mathrm{d} \Psi_{1-}}{\mathrm{dz}}
$$


Final expression for the probability of the photoemission was obtained in (Brodsky, 1973) at the assumption that electric field $\mathrm{E}$ is constant along axes z. However this is not true at the interface where the normal component of $\mathrm{E}$ is changed due to the surface charge, so that one has to consider $E=E(z)$. Using the approach of (Brodsky, 1973) with $E=E(z)$ and $\mathrm{m}=\mathrm{m}(\mathrm{z})$, one can replace Eq. (1) by

$$
\mathrm{C}_{+}(\infty)=\frac{|\mathrm{e}| \mathrm{m}}{\mathrm{W}_{1}(\hbar \omega)^{2}} \int_{-\infty}^{\infty} \frac{\mathrm{dz}}{\mathrm{m}}\left(\mathrm{c}_{\mathrm{V}}+\mathrm{c}_{\mathrm{E}}+\mathrm{c}_{\mathrm{m}}\right)
$$

where $c_{V}, c_{E}$ and $c_{m}$ describes the photoemission taking into account jumps in the potential, in the electric field and in the effective mass of the electron in the interface, respectively,

$$
\begin{gathered}
\mathrm{c}_{\mathrm{V}}=-\mathrm{EV}^{\prime} \Psi_{0} \Psi_{1-}, \quad \mathrm{c}_{\mathrm{E}}=\mathrm{E}^{\prime}\left[\frac{\hbar^{2}}{2 \mathrm{~m}} \Psi_{0}^{\prime} \Psi_{1-}^{\prime}+\left(\mathrm{E}_{0}-\mathrm{V}+\frac{\hbar \omega}{2}\right) \Psi_{0} \Psi_{1-}\right] \\
\mathrm{c}_{\mathrm{m}}=-\frac{\mathrm{Em}^{\prime}}{\mathrm{m}}\left(\mathrm{E}_{0}-\mathrm{V}+\frac{\hbar \omega}{2}\right) \Psi_{0} \Psi_{1-}
\end{gathered}
$$

Eq.(2) is integrated below for the case of step functions $V(z), E(z)$ and $m(z)$ with the step at $z=0$. For such functions $V^{\prime}=V \delta(z), E^{\prime}=\left(E_{+}-E_{-}\right) \delta(z), m^{\prime}=\left(m_{0}-m\right) \delta(z)$, where $E_{ \pm}$, are values of $E$ to the right and to the left regions from $z=0$ respectively; $m$ and $m_{0}$ are effective electron masses in the metal and outside it, respectively. Quantities $V(0)=V / 2$, $\mathrm{E}(0)=\left(\mathrm{E}_{+}+\mathrm{E}_{-}\right) / 2, \mathrm{~m}(0)=\left(\mathrm{m}_{0}+\mathrm{m}\right) / 2, \Psi^{\prime} \equiv \mathrm{d} \Psi / \mathrm{dz}$.

\subsection{Wave functions at the absence of perturbation}

Let us find wave functions of the electron moving perpendicular to the interface between the metal and the environment, the interface is described by the step potential barrier, see Fig. 2 .

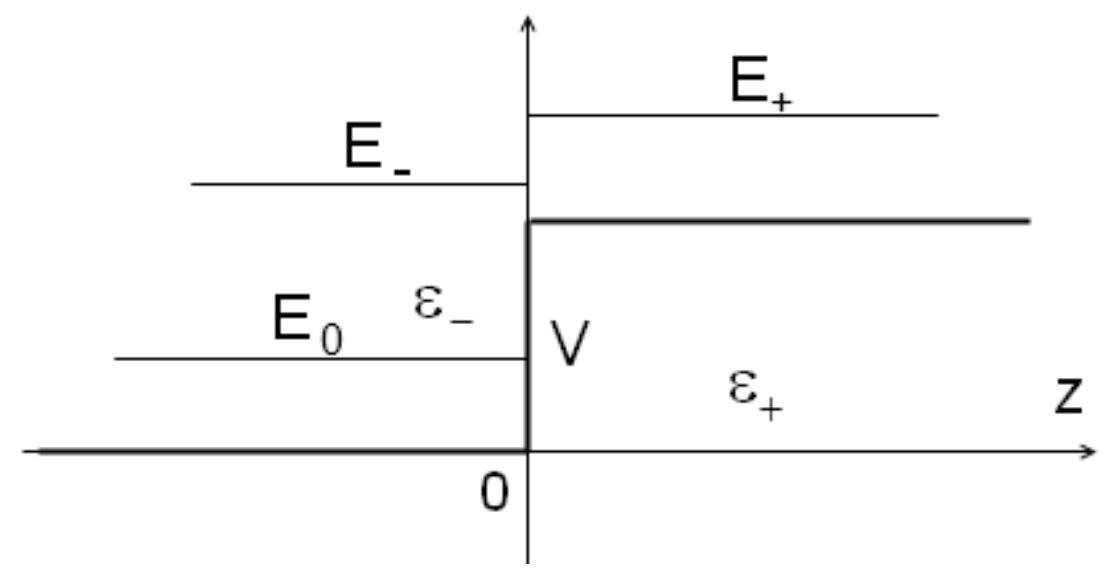

Fig. 2. Step potential barrier. The break in the electric field at $z=0$ is shown where the dielectric function $\varepsilon_{-}$of the metal is changed to the dielectric function $\varepsilon_{+}$of the environment. 
Electron of the charge e and the effective mass $m$ at $z<0$ and $m_{0}$ at $z>0$ moves in the step potential

$$
V(z)=\left\{\begin{array}{cc}
0 & z<0 \\
V / 2 & z=0 \\
V & z>0
\end{array}\right.
$$

Hamiltonian of the electron is $\mathrm{H}_{2} \mathrm{H}_{-}$at $\mathrm{z}<0$ and $\mathrm{H}=\mathrm{H}_{+}$at $\mathrm{z}>0$,

$$
\mathrm{H}_{-}=-\frac{\hbar^{2}}{2 \mathrm{~m}} \frac{\mathrm{d}^{2}}{\mathrm{dz}^{2}}, \quad \mathrm{H}_{+}=-\frac{\hbar^{2}}{2 \mathrm{~m}_{0}} \frac{\mathrm{d}^{2}}{\mathrm{dz}^{2}}+\mathrm{V}
$$

We solve Schrödinger equation $\mathrm{i} \hbar\left(\partial \bar{\Psi}_{0+} / \partial \mathrm{t}\right)=\mathrm{H}_{+} \bar{\Psi}_{0_{+}}$and find the wave function $\bar{\Psi}_{0+}=\Psi_{0} \exp \left[-i\left(E_{0} / \hbar\right) t\right]$ of the state of the electron with total energy $\mathrm{E}_{0}<\mathrm{V}$, this electron falls on the barrier and is reflected from it,

$$
\Psi_{0}=\left[\exp \left(\mathrm{ik}_{0} \mathrm{z}\right)+\mathrm{A}_{0} \exp \left(-\mathrm{ik}_{0} \mathrm{z}\right)\right]_{\mathrm{z}<0}+\left[\mathrm{B}_{0} \exp \left(\mathrm{ik}_{0} \mathrm{z}\right)\right]_{\mathrm{z}>0},
$$

here wave numbers

$$
\mathrm{k}_{0}=\frac{1}{\hbar}\left(2 \mathrm{mE}_{0}\right)^{1 / 2}, \quad \tilde{\mathrm{k}}_{0}=\frac{1}{\hbar}\left[2 \mathrm{~m}_{0}\left(\mathrm{E}_{0}-\mathrm{V}\right)\right]^{1 / 2}
$$

Because $V>E_{0}$, the value $\left(E_{0}-V\right)^{1 / 2}=i\left(V-E_{0}\right)^{1 / 2}$ is purely imaginary. The wave function $\Psi_{0}$ is normalized such that the coefficient in front of the term $\exp \left(\mathrm{ik}_{0} \mathrm{z}\right)$ describing initial electron state at $z=-\infty$ is 1 . Coefficients $A_{0}$ and $B_{0}$ in Eq.(6) are determined from conditions of regularity at $\mathrm{z}=0$

$$
\Psi_{0}(\mathrm{z}=-0)=\Psi_{0}(\mathrm{z}=+0), \quad \mathrm{m}^{-1}\left(\partial \Psi_{0} / \partial \mathrm{z}\right)_{\mathrm{z}=-0}=\mathrm{m}_{0}^{-1}\left(\partial \Psi_{0} / \partial \mathrm{z}\right)_{\mathrm{z}=+0},
$$

Eqs.(8) are equivalent to $1+\mathrm{A}_{0}=\mathrm{B}_{0}$ and $1-\mathrm{A}_{0}=\theta_{0} \mathrm{~B}_{0}$, so that

$$
A_{0}=\frac{1-\theta_{0}}{1+\theta_{0}}, \quad B_{0}=\frac{2}{1+\theta_{0}}, \quad \theta_{0}=\left[\left(m / m_{0}\right)\left(1-V / E_{0}\right)\right]^{1 / 2}
$$

Similar way we find wave functions $\bar{\Psi}_{1 \pm}=\Psi_{1 \pm} \exp \left[-\mathrm{i}\left(\mathrm{E}_{1} / \hbar\right) \mathrm{t}\right]$ of the electron state with the energy $E_{1}>V$, where

$$
\begin{aligned}
& \Psi_{1+}=\left[\mathrm{A}_{1+} \exp \left(\mathrm{ik}_{1} \mathrm{z}\right)+\mathrm{B}_{1+} \exp \left(-\mathrm{ik}_{1} \mathrm{z}\right)\right]_{\mathrm{z}<0}+\exp \left(\mathrm{ik}_{1} \mathrm{z}\right)_{\mathrm{z}>0}, \\
& \Psi_{1-}=\left[\mathrm{A}_{1-} \exp \left(\mathrm{i}_{1} \mathrm{z}\right)+\mathrm{B}_{1-} \exp \left(-\mathrm{i}_{1} \mathrm{z}\right)\right]_{\mathrm{z}>0}+\exp \left(-\mathrm{ik}_{1} \mathrm{z}\right)_{\mathrm{z}<0},
\end{aligned}
$$

and real wave numbers

$$
\mathrm{k}_{1}=\frac{1}{\hbar}\left(2 \mathrm{mE}_{1}\right)^{1 / 2}, \quad \tilde{\mathrm{k}}_{1}=\frac{1}{\hbar}\left[2 \mathrm{~m}_{0}\left(\mathrm{E}_{1}-\mathrm{V}\right)\right]^{1 / 2}
$$


Coefficients $\mathrm{A}_{1 \pm}$ and $\mathrm{B}_{1 \pm}$ are determined from conditions of regularity of the wave function at $\mathrm{z}=0$, analogous to Eq. (8), which leads to equations $A_{1 \pm}+B_{1 \pm}=1, A_{1+}-B_{1+}=\theta_{1}$, and $\theta_{1}\left(B_{1-}-A_{1-}\right)=1$, where $\theta_{1}=\left[\left(m / m_{0}\right)\left(1-V / E_{1}\right)\right]^{1 / 2}$. One can find that

$$
\mathrm{A}_{1+}=\left(1+\theta_{1}\right) / 2, \quad \mathrm{~B}_{1+}=\left(1-\theta_{1}\right) / 2, \quad \mathrm{~A}_{1-}=\left(\theta_{1}-1\right) / 2 \theta_{1}, \quad \mathrm{~B}_{1-}=\left(1+\theta_{1}\right) / 2 \theta_{1}
$$

Wave functions Eqs. (10) and (11) make the fundamental set of solutions of Schrödinger equation with Hamiltonian (5).

\subsection{Expression for probability amplitude of photoemission}

We follow the procedure of (Brodsky, 1973) and consider the monochromatic EMF of frequency $\omega$ as a perturbation

$$
\hat{\mathrm{U}}_{\text {pert }}(\mathrm{z}, \mathrm{d} / \mathrm{dz}) \cos (\omega \mathrm{t})=(1 / 2) \hat{\mathrm{U}}_{\text {pert }}(\mathrm{z}, \mathrm{d} / \mathrm{dz})\left(\mathrm{e}^{-\mathrm{i} \omega \mathrm{t}}+\mathrm{e}^{\mathrm{i} \omega \mathrm{t}}\right)
$$

for the motion of the electron in Hamiltonian Eq. (5). If we apply operator $\hat{U}_{\text {pert }}$ to the wave function $\Psi_{\mathrm{i}}, i=0,1 \pm$ of the state of the electron we obtain

$$
\hat{\mathrm{U}}_{\text {pert }} \Psi_{\mathrm{i}}=\frac{\mathrm{i} \hbar \mathrm{e}}{2 \mathrm{c}}\left[\frac{\mathrm{d}}{\mathrm{dz}}\left(\frac{\mathrm{A}}{\mathrm{m}} \Psi_{\mathrm{i}}\right)+\frac{\mathrm{A}}{\mathrm{m}} \frac{\mathrm{d} \Psi_{\mathrm{i}}}{\mathrm{dz}}\right]+\mathrm{e} \varphi \Psi_{\mathrm{i}^{\prime}}
$$

Where e is (negative) electron charge, $\mathrm{c}$ is the speed of light in vacuum, A is z-component of vector potential of EMF in the medium, $\varphi$ is scalar potential of EMF. Calculations with wave functions (10) and (11) lead to expression

$$
\left.\frac{\mathrm{W}_{1}}{\mathrm{~m}}\right|_{\mathrm{z}<0}=\left.\frac{\mathrm{W}_{1}}{\mathrm{~m}_{0}}\right|_{\mathrm{z}>0}=\mathrm{i} \frac{\mathrm{k}_{1}}{\mathrm{~m}}\left(1+\theta_{1}\right) \equiv \mathrm{i}\left(\frac{\mathrm{k}_{1}}{\mathrm{~m}}+\frac{\tilde{\mathrm{k}}_{1}}{\mathrm{~m}_{0}}\right)
$$

Taking $\mathrm{z}=0$ and that $\Psi_{0}^{\prime}, \quad \Psi_{1-}^{\prime}$ have discontinuity so that $\Psi_{0,1-}^{\prime}(0)=(1 / 2)\left[\Psi_{0,1-}^{\prime}(-0)+\Psi_{0,1-}^{\prime}(+0)\right]$ we find

$$
\Psi_{0} \Psi_{1-}=\frac{2}{1+\theta_{0}}, \quad \Psi_{0}^{\prime} \Psi_{1-}^{\prime}=\frac{2 \tilde{\mathrm{k}}_{0} \mathrm{k}_{1} \overline{\mathrm{m}}^{2}}{\mathrm{~m}_{0} \mathrm{~m}\left(1+\theta_{0}\right)},
$$

Here and below $\overline{\mathrm{m}}=\left(\mathrm{m}_{0}+\mathrm{m}\right) / 2, \Delta \mathrm{m}=\mathrm{m}_{0}-\mathrm{m}, \overline{\mathrm{E}}=\left(\mathrm{E}_{+}+\mathrm{E}_{-}\right) / 2, \Delta \mathrm{E}=\mathrm{E}_{+}-\mathrm{E}_{-}$. Inserting Eqs.(14) and (15) into Eq.(2) and proceeding some calculations we find explicit expression for the probability amplitude of the photoemission

$$
C_{+}(\infty)=\frac{2|e| m}{i k_{1} \bar{m}(\hbar \omega)^{2}\left(1+\theta_{0}\right)\left(1+\theta_{1}\right)}\left\{-V \bar{E}+\frac{\Delta E}{2}\left[E_{1}^{1 / 2}+\left(E_{0}-V\right)^{1 / 2}\right]^{2}-\frac{\bar{E} \Delta m}{2 \bar{m}}\left(E_{0}+E_{1}-V\right)\right\}
$$

Now we express Eq.(16) through the variable $x=\left(\hbar \mathrm{k}_{0}\right)^{2} /(2 \mathrm{mV}) \equiv \mathrm{E}_{0} / \mathrm{V}$. Taking into account that $\mathrm{k}_{1}=(\sqrt{2 \mathrm{mV}} / \hbar)(\mathrm{x}+\hbar \omega / \mathrm{V})^{1 / 2}, \quad \theta_{0}=\left[\left(\mathrm{m} / \mathrm{m}_{0}\right)(1 / \mathrm{x}-1)\right]^{1 / 2}$, $\theta_{1}=\left\{\left(\mathrm{m} / \mathrm{m}_{0}\right)\left[1-(\mathrm{x}+\hbar \omega / \mathrm{V})^{-1}\right]\right\}^{1 / 2}$ we find final expression for the probability of the photoemission 


$$
\left|\mathrm{C}_{+}(\infty)\right|^{2}=\mathrm{C}_{0} \mathrm{U}(\mathrm{x})\left|\mathrm{K}_{\mathrm{dis}}(\mathrm{x})\right|^{2},
$$

where the dimensionless coefficient

$$
\begin{gathered}
\mathrm{C}_{0}=\frac{2|\mathrm{e}|^{2}\left|\mathrm{E}_{-}\right|^{2} \mathrm{~V}}{\mathrm{~m} \hbar^{2} \omega^{4}}, \\
\mathrm{U}(\mathrm{x})=\frac{4 \mathrm{r}_{\mathrm{m}}^{2}}{\left(\mathrm{r}_{\mathrm{m}}+1\right)^{2}} \times \frac{\mathrm{x}}{\left[\mathrm{x}+\mathrm{r}_{\mathrm{m}}(1-\mathrm{x})\right]\left\{(\mathrm{x}+\hbar \omega / \mathrm{V})^{1 / 2}+\left[\mathrm{r}_{\mathrm{m}}(\mathrm{x}+\hbar \omega / \mathrm{V}-1)\right]^{1 / 2}\right\}^{2}}, \\
\mathrm{r}_{\mathrm{m}}=\mathrm{m} / \mathrm{m}_{0} \text { and } \\
\mathrm{K}_{\mathrm{dis}}(\mathrm{x})=\frac{1}{2}\left(1+\frac{\varepsilon_{-}}{\varepsilon_{+}}\right)\left[1+\frac{1-\mathrm{r}_{\mathrm{m}}}{1+\mathrm{r}_{\mathrm{m}}}\left(2 \mathrm{x}+\frac{\hbar \omega}{\mathrm{V}}-1\right)\right]+\frac{1}{2}\left(1-\frac{\varepsilon_{-}}{\varepsilon_{+}}\right)\left[\left(\mathrm{x}+\frac{\hbar \omega}{\mathrm{V}}\right)^{1 / 2}+\mathrm{i}(1-\mathrm{x})^{1 / 2}\right]^{2}
\end{gathered}
$$

Factor $K_{\text {dis }}$ describes both the influence of the break of EMF and the break of the effective electron mass at the interface at $z=0$; however Eq.(19) for $U(x)$ also depends on the effective electron mass. In order to came back to the case of (Brodsky, 1973), where jumps of EMF and effective mass at the interface did not taken into account, one can set $\mathrm{r}_{\mathrm{m}}=1$ and $\varepsilon_{-}=\varepsilon_{+}$in Eqs., (19), (20) which leads to $K_{\text {dis }}(x)=1$ and

$$
\mathrm{U}(\mathrm{x})=\frac{\mathrm{x}}{\left[(\mathrm{x}+\hbar \omega / \mathrm{V})^{1 / 2}+(\mathrm{x}+\hbar \omega / \mathrm{V}-1)^{1 / 2}\right]^{2}}
$$

Thus the probability of the photoemission of the electron in the case of the step potential in the interface and with account for the jumps of the electron effective mass and EMF in the interface is determined by Eq. (17), where $C_{0}$ is given by Eq. (18) and expressions for $U(x)$ and $K_{\text {dis }}(x)$ are determined by Eqs. (19) and (20), respectively. These expressions will be used below for calculation of the cross-section of photoemission from the nano-particle.

\section{Cross-section of photoemission from nano-particle for step potential.}

\subsection{Expression for cross-section of photoemission}

Cross-section $\sigma_{\text {ph-em }}$ of photoemission from the nano-particle is, by definition,

$$
\sigma_{\text {ph-em }}=\frac{J_{\text {ph-em }}}{I},
$$

where $\mathrm{J}_{\mathrm{ph}-\mathrm{em}}$ is the total photocurrent from the nanoparticle in electrons per second, I is the intensity of the external monochromatic EMF causing photoemission in photons through $\mathrm{cm}^{2}$ per second. Photocurrent from the nanoparticle is

$$
\mathrm{J}_{\text {ph-em }}=\int_{\text {surface }} \mathrm{jds}=\int_{\text {surface }} \mathrm{j}(\theta, \varphi, \mathrm{r}) \mathrm{rdr} \sin \theta \mathrm{d} \theta \mathrm{d} \varphi,
$$

where $\mathrm{j}$ is the photocurrent density in electrons through $\mathrm{cm}^{2}$ per sec, the photocurrent is normal to the surface of the nanoparticle in the point of the surface determined by polar 
angle $\theta$ azimuth angle $\varphi$ and by the distance $\mathrm{r}$ from zero of coordinate system to the surface of the nanoparticle, see Fig.3.

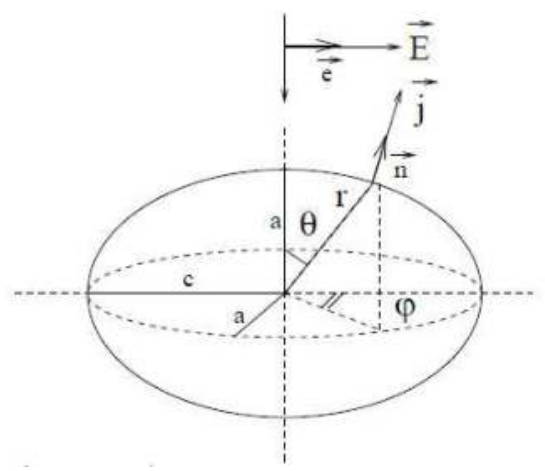

Fig. 3. The density $\mathrm{j}$ of the photocurrent in the point of the surface determined by angles $\theta$ and $\varphi$ for spheroid nanoparticle excited by the external electric field of the amplitude E. The length of two semi-axes of the particle are the same, the length of the third semi-axes is c.

Now we came from the one-dimensional model of motion of electron to the threedimensional model. Then in accordance with Eq. (2.30) of (Brodsky, 1973) we can write the photocurrent density dj of electrons with the energy in the interval $E_{0} \div E_{0}+\mathrm{dE}_{0}$ as

$$
\mathrm{dj}=\frac{\hbar \tilde{\mathrm{k}}_{1 \mathrm{z}}}{\mathrm{m}}\left|\mathrm{C}_{+}\right|^{2} \Theta\left[\mathrm{k}_{0 \mathrm{z}}^{2}+\left(2 \mathrm{~m} / \hbar^{2}\right)(\hbar \omega-\mathrm{V})\right] \mathrm{dn}_{0},
$$

where $\hbar \tilde{\mathrm{k}}_{1 \mathrm{z}} / \mathrm{m}$ is the speed of such electrons above the interface barrier, $\mathrm{dn}_{0} \equiv 2 \mathrm{f}_{\mathrm{F}}\left(\mathrm{k}_{0}\right) \mathrm{dk}_{0 \mathrm{x}} \mathrm{dk}_{0 \mathrm{y}} \mathrm{dk}_{0 \mathrm{z}} /(2 \pi)^{3}$ is the number of such electrons,

$$
\mathrm{f}_{\mathrm{F}}\left(\mathrm{k}_{0}\right)=\left[1+\exp \left\{\left[\left(\hbar \mathrm{k}_{0}\right)^{2} /(2 \mathrm{~m})-\varepsilon_{\mathrm{F}}\right] / \mathrm{k}_{\mathrm{B}} \mathrm{T}\right\}\right]^{-1}
$$

is Fermi distribution function, $\mathrm{k}_{0}$ is the wave vector of the electron before absorption of the photon, $\mathrm{k}_{0}^{2}=\mathrm{k}_{0 \mathrm{x}}^{2}+\mathrm{k}_{0 \mathrm{y}}^{2}+\mathrm{k}_{0 \mathrm{z}}^{2}, \mathrm{k}_{0 \mathrm{x}, \mathrm{y}}$ - components of the wave vector parallel to the surface of the nanoparticle, $\varepsilon_{F}$ is Fermi energy of the metal of the nanoparticle $\mathrm{k}_{\mathrm{B}}$ is Boltzmann constant, $\mathrm{T}$ is the temperature. Because of $2 \mathrm{mE}_{0} / \hbar^{2}=\mathrm{k}_{0}^{2}$ then for the step potential

$$
\tilde{\mathrm{k}}_{1 \mathrm{z}}=\sqrt{\left(2 \mathrm{~m} / \hbar^{2}\right)\left(\mathrm{E}_{0}+\hbar \omega-\mathrm{V}\right)-\left(\mathrm{k}_{0 \mathrm{x}}^{2}+\mathrm{k}_{0 \mathrm{y}}^{2}\right)} \equiv \sqrt{\mathrm{k}_{0 \mathrm{z}}^{2}+\left(2 \mathrm{~m} / \hbar^{2}\right)(\hbar \omega-\mathrm{V})}
$$

is z-component of the wave vector of the electron above the barrier (it was denoted as $\tilde{\mathrm{k}}_{1}$ in one-dimentional case), $\mathrm{k}_{0 \mathrm{z}}$ is the component of the wave vector of the electron inside the nanoparticle perpendicular to the interface, $\left|C_{+}\right|^{2} \equiv\left|C_{+}(\infty)\right|^{2}$ is the probability of photoemission and $\Theta$ is theta-function. The density $j$ of the photocurrent of electrons of all energies is

$$
\mathrm{j}=\frac{2}{(2 \pi)^{3}} \int \mathrm{dk}_{0 \mathrm{x}} \mathrm{dk}_{0 \mathrm{y}} \mathrm{dk}_{0 \mathrm{z}} \mathrm{f}_{\mathrm{F}}\left(\overrightarrow{\mathrm{k}}_{0}\right) \frac{\hbar \tilde{\mathrm{k}}_{1 \mathrm{z}}}{\mathrm{m}}\left|\mathrm{C}_{+}\right|^{2} \Theta\left[\mathrm{k}_{0 \mathrm{z}}^{2}+\left(2 \mathrm{~m} / \hbar^{2}\right)(\hbar \omega-\mathrm{V})\right]
$$


Taking into account that only $\mathrm{f}_{\mathrm{F}}$ depends on $\mathrm{k}_{0 \mathrm{x}, \mathrm{y}}$ in Eq.(25) one can take there the integral over $\mathrm{dk}_{0 \mathrm{x}} \mathrm{dk} \mathrm{k}_{0 \mathrm{y}}$ with replacement $\mathrm{k}_{0 \mathrm{x}}^{2}+\mathrm{k}_{0 \mathrm{y}}^{2}=\tilde{\mathrm{n}}^{2}$ and using $\int_{0}^{\infty} \mathrm{dx}\left(1+\mathrm{e}^{\mathrm{x}} / \mathrm{b}\right)^{-1}=\ln (1+\mathrm{b})$, so that

$$
\begin{gathered}
\int \mathrm{dk}_{0 \mathrm{x}} \mathrm{dk}_{0 \mathrm{y}} \mathrm{f}_{\mathrm{F}}\left(\overrightarrow{\mathrm{k}}_{0}\right)=\pi \int_{0}^{\infty} \frac{\mathrm{d} \rho^{2}}{1+\exp \left\{\left[\hbar^{2}\left(\rho^{2}+\mathrm{k}_{0 \mathrm{z}}^{2}\right) /(2 \mathrm{~m})-\varepsilon_{\mathrm{F}}\right] / \mathrm{k}_{\mathrm{B}} \mathrm{T}\right\}}= \\
\frac{2 \pi \mathrm{mk}_{\mathrm{B}} \mathrm{T}}{\hbar^{2}} \ln \left\{1+\mathrm{e}^{\left.\left[\varepsilon_{\mathrm{F}}-\hbar^{2} \mathrm{k}_{0 \mathrm{z}}^{2} /(2 \mathrm{~m})\right] /\left(\mathrm{k}_{\mathrm{B}} \mathrm{T}\right)\right\}}\right.
\end{gathered}
$$

Inserting Eqs. (23), (24), (26) into Eq. (25) and taking into account that according with Eqs. (17), (18) $\left|C_{+}\right|^{2} \sim\left|E_{-}\right|^{2}$, we obtain the density of the photoemission current in some point of the surface of the nanoparticle

$$
\begin{gathered}
\mathrm{j}=\mathrm{C}_{\text {emission }}\left|\mathrm{E}_{-}\right|^{2}, \\
\mathrm{C}_{\text {emission }}=\frac{|\mathrm{e}|^{2} \mathrm{k}_{\mathrm{B}} \mathrm{TV}^{2}}{\pi^{2} \hbar^{5} \omega^{4}} \int_{0,1-\mathrm{h} \omega / \mathrm{V}}^{1} \mathrm{dx}[1+(\hbar \omega / \mathrm{V}-1) / \mathrm{x}]^{1 / 2} \ln \left(1+\mathrm{e}^{\frac{\varepsilon_{\mathrm{F}}-\mathrm{Vx}}{\mathrm{k}_{\mathrm{B}} \mathrm{T}}} \mathrm{U}(\mathrm{x})\left|\mathrm{K}_{\text {dis }}(\mathrm{x})\right|^{2},\right.
\end{gathered}
$$

where the low limit of the integration is 0 if $\hbar \omega>V$ and it is $1-\hbar \omega / V$ if $\hbar \omega<V ; x<1$ due to $\mathrm{E}_{0}<\mathrm{V} ; \mathrm{U}(\mathrm{x})$ and $\mathrm{K}_{\text {dis }}(\mathrm{x})$ are determined by Eqs. (19) and (20), respectively. If we neglect by the thermal excitation of electrons above Fermi surface, i.e. take in Eq. (27) the limit $\mathrm{T} \rightarrow 0$ then, using $\mathrm{e}^{\left(\varepsilon_{\mathrm{F}}-\mathrm{Vx}\right) /\left(\mathrm{k}_{\mathrm{B}} \mathrm{T}\right)} \rightarrow \infty$, we write instead of Eq. (27)

$$
\mathrm{C}_{\text {emission }}=\frac{|\mathrm{e}|^{2}}{\pi^{2}} \frac{\mathrm{V}^{3}}{\hbar^{5} \omega^{4}} \int_{0,1-\hbar \omega / \mathrm{V}}^{\varepsilon_{\mathrm{E}} / \mathrm{V}} \mathrm{dx}[1+(\hbar \omega / \mathrm{V}-1) / \mathrm{x}]^{1 / 2}\left(\varepsilon_{\mathrm{F}} / \mathrm{V}-\mathrm{x}\right) \mathrm{U}(\mathrm{x})\left|\mathrm{K}_{\mathrm{dis}}(\mathrm{x})\right|^{2}
$$

It must be here that $\hbar \omega>V-\varepsilon_{F}$ and the low limit of integration is 0 if $\hbar \omega>V$. Thus the photocurrent from the nanoparticle is

$$
\mathrm{J}_{\text {ph-em }}=\mathrm{C}_{\text {emission }} \int_{\text {surface }}\left|\mathrm{E}_{-}\right|^{2} \mathrm{ds},
$$

where the integral is taken over the surface of the nanoparticle and $\mathrm{C}_{\text {emission }}$ does not depend on the point on the surface; normal component of the field $E_{-}=\left(\vec{E}_{i n t} \vec{n}\right)$, where $\vec{E}_{i n t}$ is the field inside the particle, $\vec{n}$ is the unit vector normal to the surface. Components of EMF tangential to the surface have no influence to the photoemission. In principle, the motion of the electron along of the surface of the nanoparticle depends on tangential components of EMF and, therefore, has the influence to the distribution function of electrons. However the EMF at the photoemission is relatively weak, so that such influence is negligibly small with the respect, for example, to the heating of the particle at the absorption of EMF. EMF inside the nanoparticle is related with external EMF $\vec{E}$ incident to the nanoparticle by the relation $\vec{E}_{\text {int }}=\hat{F}(\vec{r}) \vec{E}$, where $\hat{F}(\vec{r})$ is tensor. Spheroidal nanoparticles considered below have homogeneous EMF inside them so for such particles $\hat{\mathrm{F}}$ is constant and does not depend on $\overrightarrow{\mathrm{r}}$. For simplicity we suppose that $\overrightarrow{\mathrm{E}}$ is parallel to one of the main axes of the spheroidal particle then $\vec{E}_{\text {int }}=\overrightarrow{F E}$, where $F$ does not depend on $\vec{r}$. For non-spherical particles $F$ depends on which main axes of the particle is parallel to $\vec{E}$. Thus 


$$
\mathrm{J}_{\text {ph-em }}=\mathrm{C}_{\text {emission }}|\mathrm{F}|^{2} \mathrm{~K}_{\text {geometry }}|\mathrm{E}|^{2} \text {, }
$$

where $\mathrm{K}_{\text {geometry }}=\int_{\text {surface }}(\overrightarrow{\mathrm{n}}), \overrightarrow{\mathrm{e}}$ is unit vector parallel to the polarization of the external field, see Fig.3. Taking into account Eq. (21) and the intensity I of external EMF (in photons through $\mathrm{cm}^{2}$ per $\mathrm{sec}$ ), which is

$$
\mathrm{I}=\frac{1}{8 \pi} \frac{\mathrm{cn}+|\mathrm{E}|^{2}}{\hbar \omega}
$$

we find the cross-section of the photoemission

$$
\sigma_{\text {ph-em }}=\frac{8 \pi \hbar \omega}{\mathrm{cn}_{+}} \mathrm{C}_{\text {emission }}|\mathrm{F}|^{2} \mathrm{~K}_{\text {geometry }},
$$

where $\mathrm{c}$ is the speed of light in vacuum, $\mathrm{n}_{+}=\operatorname{Re} \sqrt{\varepsilon_{+}}$is the refractive index of the medium outside the nanoparticle.

\subsection{Parameters $\mathrm{F}$ and $\mathrm{K}_{\text {geometry }}$}

According with (Meier \& Wokaun, 1983)

$$
\mathrm{F}=\frac{1}{1+\mathrm{R}_{\mathrm{dep}}-\mathrm{iR} \mathrm{R}_{\mathrm{rad}}} \times \frac{\varepsilon_{+}}{\varepsilon_{+}+\left(\varepsilon_{-}-\varepsilon_{+}\right) \mathrm{L}}
$$

for spheroidal particles, where the second multiplier is the result of calculations in quazistatic approach,

$$
\mathrm{L}=\frac{\mathrm{r}^{2}}{2} \int_{0}^{\infty} \frac{\mathrm{du}}{\left(\mathrm{u}+\mathrm{r}^{2}\right)^{2}(\mathrm{u}+1)^{1 / 2}}
$$

aspect ratio $r=a / c$, a is the length of one of two equal semi-axes of ellipsoidal particle, $c$ is the length of the third semi-axes, see Fig.3; the second multiplier in Eq. (32) takes into account dynamic depolarization (factor $R_{\text {dep }}$ ) and radiative losses (factor $R_{\text {rad }}$ ) (Bottcher, 1952)

$$
\begin{aligned}
R_{\text {dep }}= & \frac{\varepsilon_{-}-\varepsilon_{+}}{\varepsilon_{+}+\left(\varepsilon_{-}-\varepsilon_{+}\right) L}\left(A \varepsilon_{+} y^{2}+B \varepsilon_{+}^{2} y^{4}\right), \quad R_{\text {rad }}=\frac{16 \pi^{3}}{9} \frac{n_{+}^{3}}{r}\left(\frac{a}{\lambda}\right)^{3} \frac{\varepsilon_{-}-\varepsilon_{+}}{\varepsilon_{+}+\left(\varepsilon_{-}-\varepsilon_{+}\right) L}, \\
A & =-0.4865 \mathrm{~L}-1.046 \mathrm{~L}^{2}+0.848 \mathrm{~L}^{3}, \quad B=0.01909 \mathrm{~L}+0.1999 \mathrm{~L}^{2}+0.6077 \mathrm{~L}^{3},
\end{aligned}
$$

where $y=\pi \mathrm{a} / \lambda, \lambda$ is the wavelength of EMF in vacuum. Factor $R_{\text {dep }}$ is characterized the nonhomogeneity of EMF inside the particle. Collisions of electrons with the surface of the nanoparticle lead to the deviation of the dielectric function $\varepsilon$ - of metal of nanoparticle from the dielectric function $\varepsilon_{\text {bulk }}$ of the macroscopic (bulk) piece of the same metal. This difference can be taken into account according with (Brongersma, 2007)

$$
\varepsilon_{-}=\varepsilon_{\text {bulk }}+\frac{\omega_{\mathrm{pl}}^{2}}{\omega^{2}+i \omega \gamma_{0}}-\frac{\omega_{\mathrm{pl}}^{2}}{\omega^{2}+i \omega\left(\gamma_{0}+i A \nu_{\mathrm{F}} / \mathrm{a}\right)},
$$


where $\omega_{\mathrm{pl}}$ and $\gamma_{0}$, are plasma frequency and the dumping increment, respectively, $v_{\mathrm{F}}$ is the rate of electrons near Fermi surface; $\mathrm{A}$ is constant of the order of 1 depending on the shape of the particle. According with (Meier, 1983)

$$
\mathrm{K}_{\text {geometry }}=\frac{\pi \mathrm{a}^{2}}{\mathrm{r}}\left[\frac{\mathrm{r}}{1-\mathrm{r}^{2}}+\frac{1-2 \mathrm{r}^{2}}{\left(1-\mathrm{r}^{2}\right)^{3 / 2}} \arcsin \left(1-\mathrm{r}^{2}\right)^{1 / 2}\right]
$$

Thus the cross-section of the photoemission is determined by expression (31) where coefficient $\mathrm{C}_{\text {emission }}$ is determined by Eq. (27), factors $\mathrm{F}$ and $\mathrm{K}_{\text {geometry }}$ are given by Eqs. (32) and (35), respectively. One can compare the cross-section of the photoemission with crosssections $\sigma_{\mathrm{abs}}$ and $\sigma_{\mathrm{sc}}$ of absorption and scattering of the light by nanoparticle (Meier \& Wokaun, 1983)

$$
\sigma_{\mathrm{abs}}=\frac{8 \pi^{2} \mathrm{a}^{3} \mathrm{n}_{+}}{3 \mathrm{r} \lambda} \operatorname{Ima}, \quad \sigma_{\mathrm{sc}}=\frac{128 \pi^{5} \mathrm{a}^{6} \mathrm{n}_{+}^{4}}{27 \mathrm{r}^{2}}|\mathrm{a}|^{2}, \quad \mathrm{a}=\frac{1}{1+\mathrm{R}_{\mathrm{dep}}-\mathrm{i} \mathrm{R}_{\mathrm{rad}}} \times \frac{\varepsilon_{-}-\varepsilon_{+}}{\varepsilon_{+}+\left(\varepsilon_{-}-\varepsilon_{+}\right) \mathrm{L}}
$$

Numerical estimations of the cross-sections of the photoemission from metal nanoparticles will be carried out in the next Subsection.

\section{Photoemission from gold nanoparticles into $\mathrm{Si}$}

Let us calculate the cross-section of photoemission from the spherical gold nanoparticle into p-doped Si. We chose Si as the environment of the nanoparticle because of the work function $\chi_{e}$ for electron coming from Au to p-type Si is small $\chi_{e}=0.34 \mathrm{eV}$ (Dutta, 2009). Because of Fermi energy for $\mathrm{Au} \varepsilon_{\mathrm{F}}=5.1 \mathrm{eV}$ (Dutta, 2009), the height of the barrier in Au - p$\mathrm{Si}$ interface is $\mathrm{V}=\varepsilon_{\mathrm{F}}+\chi_{\mathrm{e}}=5.44 \mathrm{eV}$. The electron effective mass in $\mathrm{Au}$ and in $\mathrm{Si}$ is, respectively $\mathrm{m}=0.992 \mathrm{~m}_{1} \approx \mathrm{m}_{1}$ and $\mathrm{m}_{0}=0.25 \mathrm{~m}_{1}$ (Kittel, 1996), where $\mathrm{m}_{1}$ is the mass of free electron in vacuum; so that $r_{m}=0.992 / 0.25=3.968$. The data for the dielectric function $\varepsilon_{\mathrm{Au}}$ of $\mathrm{Au}$ are taken from (Weber, 2002). It is convenient to write the dielectric function (34) of $\mathrm{Au}$ as a function of the wavelength $\lambda$ of EMF in vacuum:

$$
\varepsilon_{-}(\lambda)=\varepsilon_{\mathrm{Au}}(\lambda)+\left(\frac{\lambda}{\lambda_{\mathrm{p}}}\right)^{2}\left[\frac{1}{1+\mathrm{i} \lambda / \lambda_{\mathrm{f}}}-\frac{1}{1+\left(\mathrm{i} \lambda / \lambda_{\mathrm{f}}\right)\left(\mathrm{a}_{\mathrm{c}} / \mathrm{a}+1\right)}\right],
$$

we take $\lambda_{\mathrm{p}}=0.142 \mu \mathrm{m}$ and $\lambda_{\mathrm{f}}=55 \mu \mathrm{m}$, they correspond to the best approximation

$$
\varepsilon_{\mathrm{Au}}(\lambda)=12+\left(\frac{\lambda}{\lambda_{\mathrm{p}}}\right)^{2} \frac{1}{1+\mathrm{i} \lambda / \lambda_{\mathrm{f}}}
$$

in the region of $\lambda$ from 0.6 to $1.2 \mu \mathrm{m}$ where LPR of spherical $\mathrm{Au}$ nanoparticle in $\mathrm{Si}$ is located; $\mathrm{a}_{\mathrm{c}}=\mathrm{A} v_{\mathrm{f}} \lambda_{\mathrm{f}} /\left(2 \pi \mathrm{c}_{0}\right)$ is the parameter characterizing the collision of the electron with the surface of the nanoparticle, $\mathrm{A}=0.7, \mathrm{v}_{\mathrm{F}}=\left(2 \mathrm{E}_{\mathrm{F}}|\mathrm{e}| / \mathrm{m}_{0}\right)^{1 / 2} \approx 1.3 \times 10^{6} \mathrm{~m} / \mathrm{sec}$; it is supposed that effective electron mass in $\mathrm{Au}$ is equal to free electron mass. Fig. 4 shows $\mathrm{Re}$ and Im parts of $\varepsilon_{\mathrm{Au}}(\lambda)$, its approximation according with Eq.(38) is quite close to $\varepsilon_{\mathrm{Au}}(\lambda)$ and $\varepsilon_{-}(\lambda)$ found from Eq.(37) for $\mathrm{a}=10 \mathrm{~nm}$. One can see that $\operatorname{Im}\left[\varepsilon_{-}(\lambda)\right]$ is noticeably greater than 
$\operatorname{Im}\left[\varepsilon_{\mathrm{Au}}(\lambda)\right]$, which points out to the necessity of taking into account collisions of electrons with the surface of the nanoparticle. We can use approximation (Adachi, 2002) for the dielectric function $\varepsilon_{+}(\lambda)$ of $S i$

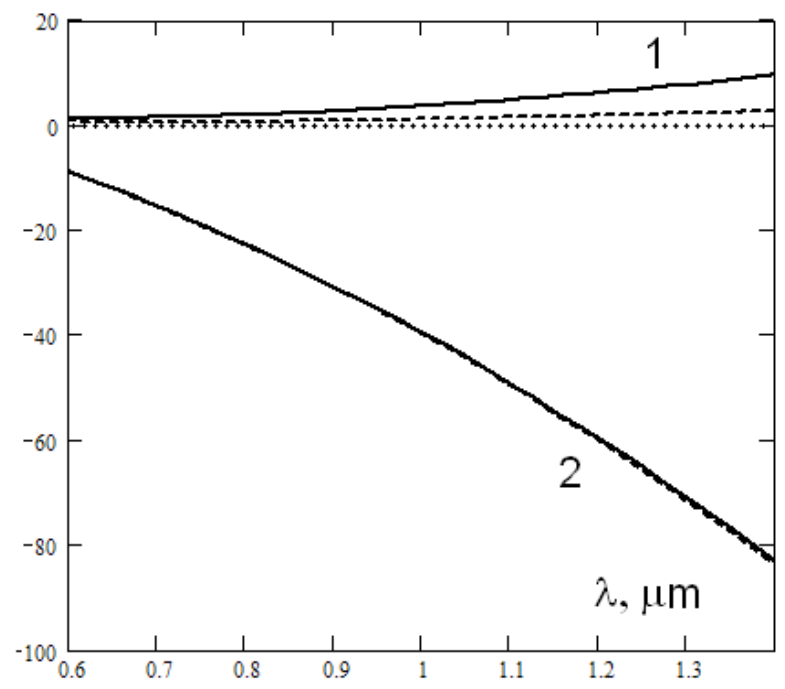

Fig. 4. Imaginary 1 and real 2 parts of the dielectric function of Au according with (Weber, 2003) are shown by dashed lines; according with Eq.(37) - by solid lines. Dotted horizontal line means 0 .

$$
\varepsilon_{+}(\lambda)=\varepsilon_{\infty}+\sum_{\mathrm{i}=1}^{3} \frac{\mathrm{C}_{\mathrm{i}}}{1-\left(\frac{1.242}{\lambda \mathrm{E}_{\mathrm{i}}}\right)^{2}-\mathrm{i} \frac{1.242}{\lambda \mathrm{E}_{\mathrm{i}}} \gamma_{\mathrm{i}}}-\mathrm{F}_{1} \chi_{1}^{-2}(\lambda) \ln \left[1-\chi_{1}^{2}(\lambda)\right]-\mathrm{F}_{2} \chi_{2}^{-2}(\lambda) \ln \frac{1-\chi_{1}^{2}(\lambda)}{1-\chi_{2}^{2}(\lambda)}
$$

where

$$
\begin{gathered}
\chi_{\mathrm{m}}(\lambda)=\left(\frac{1.242}{\lambda}+\mathrm{i} \Gamma_{\mathrm{m}}\right) \frac{1}{\mathrm{E}_{\mathrm{m}}} \text { and } \varepsilon_{\infty}=0.2, \mathrm{C}_{1}=0.77, \mathrm{C}_{2}=2.96, \mathrm{C}_{3}=0.3, \mathrm{~F}_{1}=5.22, \mathrm{~F}_{2}=4, \\
\gamma_{1}=0.05, \mathrm{Y}_{2}=0.1, \mathrm{\gamma}_{3}=0.1, \mathrm{E}_{1}=3.38, \mathrm{E}_{2}=4.27, \mathrm{E}_{3}=5.3, \Gamma_{1}=0.08, \Gamma_{2}=0.1
\end{gathered}
$$

Fig.5a shows cross-sections (36) of absorption and scaterring of Au nanoparticle in Si in $\pi \mathrm{a}^{2}$ units. One can see that the absorption cross-section is greater than $\pi \mathrm{a}^{2}$ more than one order of magnitude near $\lambda=\lambda_{\text {LPR }}=0.857 \mu \mathrm{m}$. Fig. $5 \mathrm{~b}$ shows the factor $|\mathrm{F}|^{2}$ appeared in Eq.(31), that is the factor of increase of intensity of EMF in the nanoparticle with the respect to the intensity of ENF outside the nanoparticle, F is determined by Eq.(32). At the excitation of LPR the intensity of EMF inside the nanoparticle is 150 times greater than the intensity of the external EMF outside the nanoparticle.

Cross-section $\sigma_{\mathrm{ph}-\mathrm{em}}$ of the photoemission from spherical Au nanoparticle of radius $\mathrm{a}=10$ $\mathrm{nm}$ in $\mathrm{Si}$ is shown in Fig.6a, together with $\sigma_{\mathrm{abs}}$ and $\sigma_{\mathrm{sc}}$, as function of the wavelength of 
the incident radiation, as it was found from the formula (31) with the use of Eqs. (27), (32) and (35).
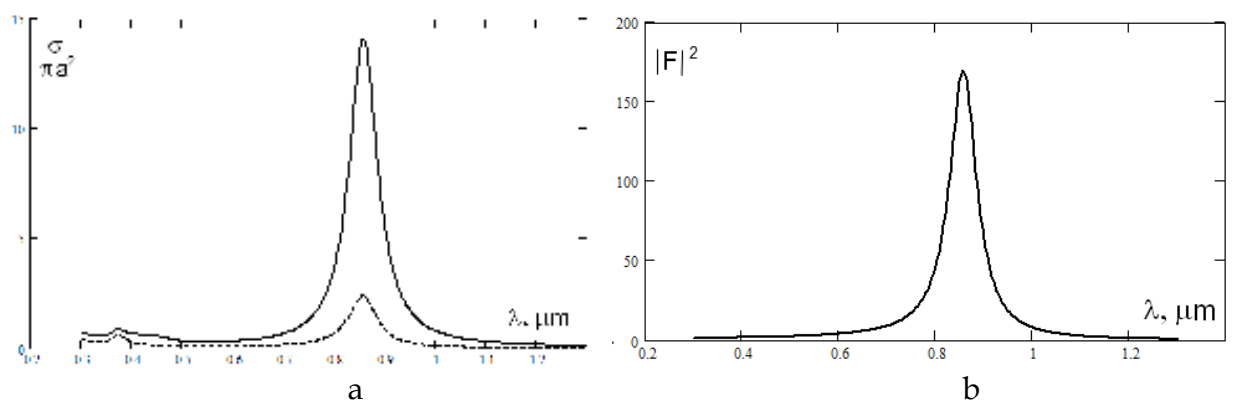

Fig. 5. (a) cross-section of absorption $\sigma_{\text {abs }}$ (solid line) and scattering $\sigma_{\mathrm{sc}}$ (dashed line) of spherical Au nanoparticle in Si. (b) The factor of the enhancement of EMF in the nanoparticle.

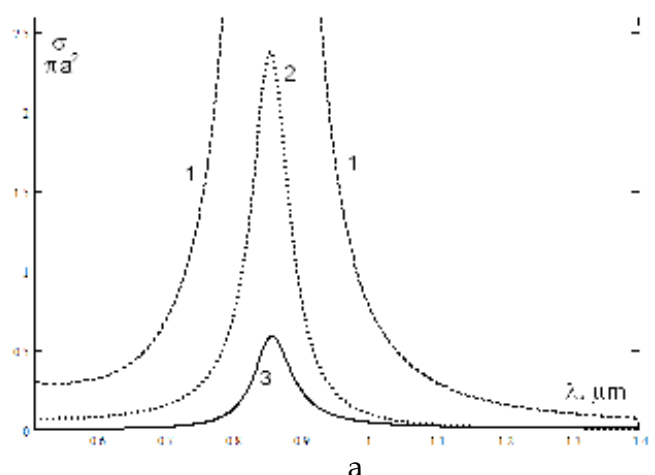

a

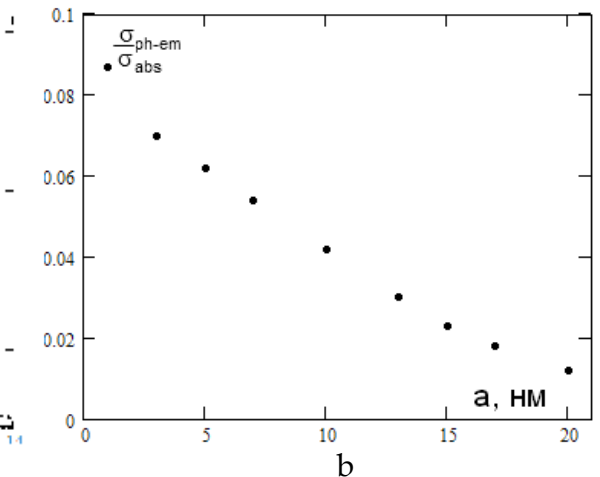

b

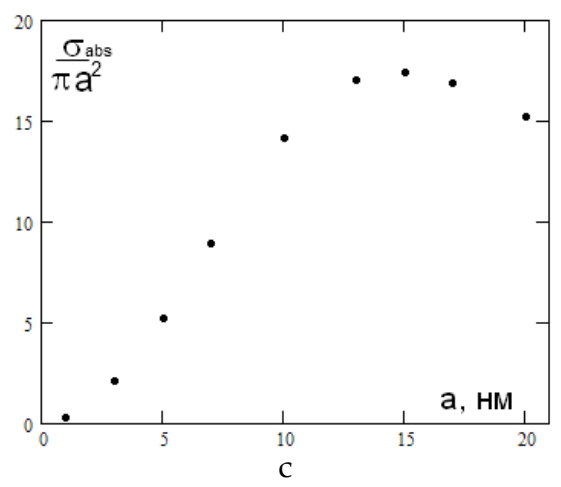

Fig. 6. (a) Cross-section of photoemission (curve 3), absorption (2) and scattering (1) in units of the geometrical cross-section $\pi \mathrm{a}^{2}$ of spherical Au nanoparticle; (b) the ratio $\sigma_{\text {ph-em }} / \sigma_{\text {abs }}$ at the maximum of LPR for various radii of spherical nanoparticles; (c) cross-section of absorption of spherical Au nanoparticle versus its radius. 
It can be seen from Fig.6a that $\sigma_{\mathrm{ph}-\mathrm{em}}$ reaches approximately the half of the geometrical cross-section $\pi \mathrm{a}^{2}$ of the nanoparticle, which is about $4.2 \%$ of the maximum value of $\sigma_{\text {abs }}$. The ratio $\sigma_{\text {ph-em }} / \sigma_{\mathrm{abs}}$ at the resonance, i.e. at $\lambda=\lambda_{\mathrm{LPR}}$, is shown in Fig.6b, it characterizes relative part of the energy absorbed by nanoparticles and converted to the photocurrent. Though this relative part is not so large (about few percents), it is much bigger than for the case of continues Au film (see below). Fig.6b shows that the ratio $\sigma_{\text {ph-em }} / \sigma_{\text {abs }}$ is decreased almost linearly from $9 \%$ to $1 \%$ at the increase of the radius of nanoparticle from 1 to $20 \mathrm{~nm}$. Thus the photoemission is relatively more effective for small particles. However the absorption cross-section itself is small at small radius a of the nanoparticle. This is because of the broadening of LPR due to collisions of electrons with the surface of the nanoparticle. Cross-section $\sigma_{\text {abs }}$ reaches the maximum, for large a it goes down because of de-phasing and radiative losses, see Fig.6b. The optimum value of the radius of the nanoparticle can be estimated from calculations of the photoemission current made below.

Collective phenomena, as the interaction of particles with each other through EMF, may be quite important at the photoemission from the ensemble of nanoparticles. Detailed description of the influence of collective phenomena on the photoemission from nanoparticles is outside the score of present study; here we restrict ourselves only by some estimation. Noting that the number of photons absorbed per unit of time in the metal film can't exceed the number of photons falls per unit of time down to the surface of the film outside, one can write $\sigma_{\text {abs }} /\left(\pi \mathrm{a}^{2}\right)<1 / \eta$ where $\eta$ is relative surface density of nanoparticles, i.e. $\sigma_{\text {abs }}$ must decrease with the increase of $\eta$ if $\sigma_{a b s}>\pi \mathrm{a}^{2}$. In practice $\sigma_{a b s}$ is decreased with $\eta$ due to the broadening of LPR caused by collective phenomena. Quite possible that the broadening of LPR leads to the decrease of the factor F and the crosssection of photoemission for narrow spectrum of LPR, when $\lambda$ is close to $\lambda_{\text {LPR }}$, however it does not mean that the photoemission from the ensemble of nanoparticles will be decreased with increase of $\eta$ at broad spectrum of EMF as, for example, the solar spectrum. Note that narrow high-quality LPRs have been predicted and observed at certain conditions in ensembles of nanoparticles (Hicks, 2005), which means that the factor F may be quite big in encembles of nanoparticles even if $\sigma_{\mathrm{abs}}$ is not large. Thus one can't say a-priori that collective phenomena allways decrease the photoemission from nanoparticles. Detailed investigation of the influence of collective phenomena on the photoemission from nanoparticles will be carried out in future, here we compare the photoemission from the layer of nanoparticles with the photoemission from the continues metal film by using formulas obtained above without taking into account collective phenomana.

We estimate now the surface density of the photoemission current. We use Eq.(27) in order to estimate the density $\mathrm{j}_{\mathrm{ph}-\mathrm{em}}$ of the photoemission current from thin continues film of $\mathrm{Au}$; in Eq.(27) E is the component of EMF normal to the surface of the metal film. For simplicity we suppose that that $\mathrm{E}_{-}=\mathrm{E}$ this way we rather overestimate the photoemission current from the continues metal film.

Suppose that we have the layer of spherical Au nanoparticles in Si with relative surface density $\eta$; we estimate the surface density of the photocurrent if the layer is illuminated by solar radiation. Normalized solar spectrum is 


$$
\mathrm{w}_{\mathrm{s}}(\lambda)=\frac{\lambda^{-4} / 0.128}{\exp (2.616 / \lambda)-1}
$$

where $\lambda$ is the wavelength in $\mu \mathrm{m}$. The surface density $\mathrm{j}_{\mathrm{ph}-\mathrm{em}}$ of the photocurrent normalized to the total intensity I of solar radiation is

$$
\frac{\mathrm{j}_{\mathrm{ph}-\mathrm{em}}}{\mathrm{I}}=\frac{\eta}{\pi \mathrm{a}^{2}} \int \mathrm{w}_{\mathrm{s}}(\lambda) \sigma_{\mathrm{ph}-\mathrm{em}}(\lambda) \mathrm{d} \lambda
$$

Fig.7a shows the ratio of the surface density of photoemission versus the radius of the nanoparticle, - from the layer of spherical Au nanoparticles to the surface density of the photoemission current from continues Au layer for $\eta=0.3$, i.e. when $30 \%$ of the surface is covered by $\mathrm{Au}$ nanoparticles. We take the spectral range of solar radiation from 0.32 to 2 $\mathrm{nm}$, with about $80 \%$ of total energy of solar radiation.

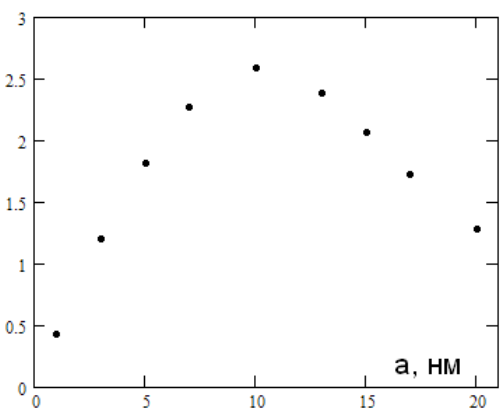

a

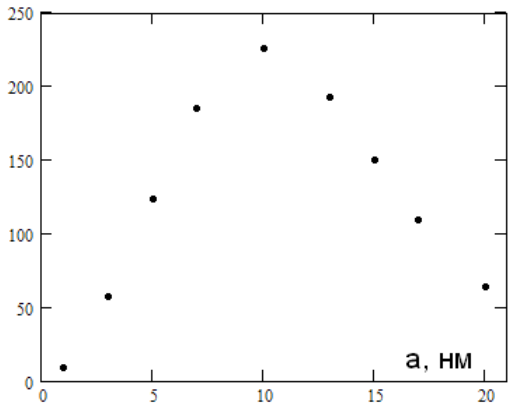

b

Fig. 7. (a) The ratio of the surface density of the photoemission current from the layer of nanoparticles with $\eta=0.3$ to the surface density of the photoemission current from continues Au layer in Si for solar radiation for different radiuses of nanoparticles; (b) the same for monochromatic EMF at LPR.

Fig.7b shows the same quantity as Fig.7a but for the monochromatic EMF at the wavelength of LPR. One can see from Fig.7a that the photoemission current from the layer of nanoparticles is several times greater than from continues metal film for the case of solar radiation. There is optimal value of radius of nanoparticles when the photocurrent has maximum. For the monochromatic EMF near LPR the photocurrent from the layer of nanoparticles exceeds two orders of magnitudes the photocurrent from continues layer, see Fig.7b. Optimum radius of nanoparticles in this case is $10 \mathrm{~nm}$. We consider here the "internal" efficiency of the photoemission from nanoparticles. The efficiency of collecting of the photocurrent into the external circuit is not considered. Estimations here do not take into account several factors as, for example, tunneling of photo-curriers through the potential barrier on the interface between the particle and the environment (Nolle, 2007); we can also note that the probability of the above barrier transition for the photo-carrier may be larger than the probability for the photo-induced carrier to leave potential well. Account for the potential barrier instead of the potential well can be done by using the approach presented 
above, it can be done analytically for step potential or numerically for more realistic smooth potentials also with an account for image forces. The photoemission from the volume of the nanoparticle may bring additional, but may be not so large, contribution to the photocurrent; it may be done following, for example, the approach of (Fowler, 1931).

In a difference with (Brodsky, 1973) here we take into account the change in the normal component of the electric field and in the electron effective mass at the interface between the metal and the environment. Let us estimate how important are these changes ("jumps") for the case of the photoemission from Au nanoparticle to p-doped Si. In order to came back to results of (Brodsky, 1973) (i.e. without "jumps") one can follow the procedure described after Eq. (20). Fig. 8 show the cross-section of photoemission neglecting by the change of the effective mass of the electron, i.e. when $r_{m}=1$, of neglecting by the change of EMF, when $\varepsilon_{-} / \varepsilon_{+}=1$, of when we neglect by both of them, when $r_{m}=\varepsilon_{-} / \varepsilon_{+}=1$. For comparison Fig.8 displays the cross-section of photoemission for $r_{\mathrm{m}} \neq 1$ and $\varepsilon_{-} / \varepsilon_{+} \neq 1$, that is the same as the curve 3 in Fig.6a.

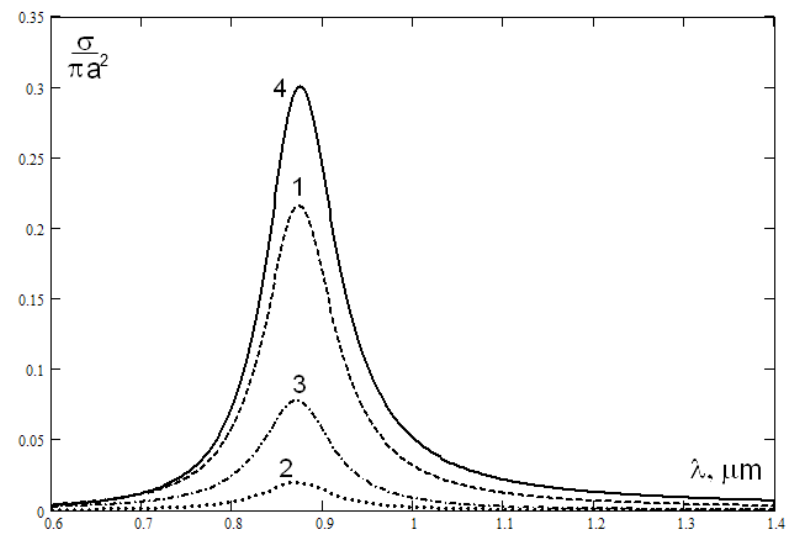

Fig. 8. Cross-sections of the photoemissions. We neglect by changes: of the effective mass of the electron at the interface (curve 1); EMF (2); the effective mass and EMF (3) that is the result of (Brodsky, 1973); all changes are taken into account (4).

According with Fig. 8 account for the jump in EMF and in the effective mass considerably changes (increases) the value of the cross-section of photoemission. It is interesting to note that the maximum value of the cross-section is reached when all jumps are taken into account. The jump in the mass, without the jump in the EMF reduces photoemission (compare curves 2 and 3 in Fig.8), the jump in the EMF without the jump in the mass increases photoemission (compare curves 1 and 3 in Fig.8), but both of them lead to the maximum in photoemission (compare curves 4 with others in Fig.8). Such "non-additive" influence of jumps of EMF and electron mass to the cross-section of photoemission is the consequence of quantum-mechanical interference. Indeed, complex terms $C_{V}, c_{E}$ and $c_{m}$ describing, respectively, the photoemission with account for jumps in the potential, EMF and the electron mass at the interface appear as linear (additive) combination in Eq. (2) for the probability amplitude $C_{+}(\infty)$ of the photoemission. However the probability 
$\mathrm{C}_{\text {emission }} \sim\left|\mathrm{C}_{+}(\infty)\right|^{2}$ appears in Eq. (31) for $\sigma_{\mathrm{ph}-\mathrm{em}}$, and noticerable quantum-mechanic interferience between contributions from $\mathrm{C}_{\mathrm{V}}, \mathrm{C}_{\mathrm{E}}$ and $\mathrm{c}_{\mathrm{m}}$ is. This interferience leads to incease of $\sigma_{\text {ph-em }}$, when all three terms are taking into account, but it leads to increase or to decrease of $\sigma_{\text {ph-em }}$ when there are only two or only one term. In practice it means that various interfaces may have quite different and unusual influence to the photoemission.

\section{Conclusion}

We calculated the probability amplitude and the cross-section of the photoemission from metal nanoparticles. It is shown that the cross-section of photoemission from metal nanoparticles is about the half of the geometrical cross-section of the nanoparticle, see Fig.6a; an example of the photoemission from Au spherical nanoparticles into p-doped $\mathrm{Si}$ is considered. It turns to be that if the surface density of nanoparticles in the layer is $\eta=0.3$ then about $15 \%$ of all photons can be converted to photoelectrons at the excitation of the localized plasmon resonance (LPR), so that $15 \%$ is the internal quantum efficiency of photoemission at $\lambda=\lambda_{\text {LPR }}$. The photoemission current from the layer of nanoparticles is two orders of magnitude greater than from continues layer of $\mathrm{Au}$ at the monochromatic EMF exciting LPR. For the case of the broad spectrum of EMF (as solar spectrum) the photoemission from nanoparticles is several times more intensive that from continues metal layer, see Fig.7, there is the optimum radius of nanoparticles corresponding to the maximum of the photoemission current. Increase of the photoemission from nanoparticles with the respect to continues metal layer is occurred due to the increase of EMF inside and near nanoparticle at the excitation of EMF (see Fig.5) and due to relatively large surface of the nanoparticle, which surface is non-parallel to the polarization of the incident EMF.

We generalize the theory of (Brodsky, 1973) of the photoemission from metals by taking into account breaks in the EMF and in the electron effective mass in the interface of the metal and environment. It is shown that such breaks may considerably change the cross-section of photoemission from metals, as one can see from the example of photoemission from $\mathrm{Au}$ particles to p-doped Si considered here.

We do not take into account the volume photo-effect, which can increase the photocurrent even more; the potential in the metal-environment interface was approximated by rectangular potential well. However in reality there is a barrier in the interface. Account for the potential barrier in the interface and for the tunneling through the barrier will lead to more increase in the photoemission current. Calculations with more complicated potentials on the interface may be proceeded by direct generalization of the approach presented above. In the future one has to take into account collective phenomena as the interaction of nanoparticles with each other through EMF, however it influences only the factor F describing the increase of EMF inside nanoparticle with the respect to EMF outside it. Approach of this work can be useful for description and study of recombination of carriers (also photoinduced carriers) on metal nanoparticles.

Results of this work can be used for creating of new high-sensitive photo detectors and photo converters of solar radiation into electric energy. There is important question about the minimum time of the photo effect related with the sensitivity of photodetectors (Schelev, 2000). It is possible, that the increase of the efficiency of the photoemission at the excitation 
of LPR lets to reduce the minimum time necessary to observe the photo effect. For example, if the femto-second laser pulse can excite LPR in the nanoparticle, then the photo detector with such nanoparticles can provide femtosecond time resolution even if the time of the photo-responce of the metal of the nanoparticle is larger than femtosecond. This way the question about shortest time of photo-response of the metal is replaced, therefore, by the question of the shortest time of the excitation of LPR.

\section{References}

Adachi, S., Mori, H., Ozaki, S. (2002). Model dielectric function for amorphous semiconductors. Physical Review B, Vol. 66, No.15, (2 October 2002), pp.(153201 [4 pages]), ISSN 1098-0121

Atwater, H. A., Polman, A. (2010). Plasmonics for improved photovoltaic devices. Nature Materials, Vol. 9, (March 2010), pp. (205-213), ISSN 1476-1122

Bergman, D. J., Stockman, M. I. (2003). Surface Plasmon Amplification by Stimulated Emission of Radiation: Quantum Generation of Coherent Surface Plasmons in Nanosystems. Physical Review Letters, Vol. 90, No. 20, (14 January 2003), pp. (027402 [4 pages]), ISSN 0031-9007

Bottcher, C. J. F. (1952). Theory of Electric Polarization, Vol. 1, Elsevier, Amsterdam

Brodsky, A.M. \& Gurevich, Yu. Ya. (1973). Theory of electron emission from metals, Nauka, Moscow, Russia

Brongersma, M. L., Kik, P. G. (Eds.). (2007). Surface Plasmon Nanophotonics, Springer-Verlag, ISBN 978-140-2043-49-9, New York, NY, US

Catchpole, K. R., Polman, A. Plasmonic solar cells. (2008). Optics Express, Vol. 16, No. 26, (22 December 2008), pp. (21793-21800), ISSN 1094-4087

Dutta, A., Mazhari, B., \& Visweswaran, G. S. (2009). Metal Semiconductor Contact. Schottky barrier height, In: Semiconductor Devices (NPTEL Online - IIT Dehli 2011), Available from:

http://nptel.iitm.ac.in/courses/Webcourse-contents/IIT-

Delhi/Semiconductor\%20Devices/index.htm

Fowler, R. H. (1931). The analysis of photoelectric sensitivity curves for clean metals at various temperatures. Physical Review, Vol.38, No.1, (July 1931), pp. (45-56), ISSN 1050-2947

Greffet, J.-J. (2005). Nanoantennas for Light Emission. Science, Vol. 308, No. 5728, (10 June 2005), pp. (1561-1563), ISSN 0036-8075

Hetterich, J., Bastian, G., Gippius, N.A., Tikhodeev, S.G., von Plessen, G., \& Lemmer, U. (2007). Optimized Design of Plasmonic MSM Photodetector IEEE Journal of Quantum Electronics, Vol. 43, No. 10, (October 2007), pp. (855-859), ISSN 0018-9197

Hicks, E. M., Zou, S., Schatz, G.C., Spears, K.G., Van Duyne, R.P., Gunnarsson, L., Rindzevicius, T., Kasemo, B., \& Käll, M. (2005). Controlling Plasmon Line Shapes through Diffractive Coupling in Linear Arrays of Cylindrical Nanoparticles Fabricated by Electron Beam Lithography Nano Letters, Vol. 5, No. 6, (18 May 2005), pp.(1065-1070), ISSN: 1530-6984

Homola, J., Yee S. S., \& Gauglitz, G. (1999). Surface plasmon resonance sensors: review. Sensors and Actuators B, Vol. 54, No. 1-2, (25 January 1999), pp. (3-15), ISSN 09254005 
Hövel, H., Fritz, S., Hilger, A., \& Kreibig, U. (1993). Width of cluster plasmon resonances: Bulk dielectric functions and chemical interface damping. Physical Review B, Vol. 48, No. 24, (December 1993), pp. (18178-18188), ISSN 1098-0121

Khlebtsov, N. G. (2008). Optics and biophotonics of nanoparticles with a plasmon resonance. Quantum electronics, Vol. 38, No. 6, (June 2008), pp. (504-529), ISSN 1063-7818

Kittel, Ch. (1996). Introduction to Solid State Physics, Jon Wiley \& Sons inc., London, New York, ISBN 978-047-1111-81-8

Klimov, V. V., (2003). Spontaneous atomic radiation in the presence of nanobodies. PhysicsUspekhi, Vol. 46, No. 10, (September 2003), pp. (979-984), ISSN 0042-1294

Klimov, V.V., (2009). Nanoplasmonics, Fizmatlit, ISBN 978-5-9221-1030-3, Moscow, Russia

Kneipp, K., Moskovits, M., \& Kneipp, H. (Eds.). Topics in Applied Physics Vol. 103, SpringerVerlag, ISBN 978-3-540-33566-5 , Berlin, Heidelberg, New York

Landau, L. D., Lifshitz, L. M. (1997). Quantum Mechanics: Vol 3, Butterworth-Heinemann, ISBN 978-0-750-63539-4, International edition

Maier, S. A. (2007). Plasmonics: Fundamentals and Applications, Springer-Verlag New York Inc., ISBN 978-038-7331-50-8, New York, NY, US

Meier, M., Wokaun, A. (1983). Enhanced fields on large metal particles: dynamic depolarization. Optics Letters, Vol.8, No.11, (1 November 1983), pp. (581-583), ISSN 0146-9592

Monestier, F., Simona, J.-J., , Torchioa, P., Escoubasa, L., Florya, F., Baillyb, S., Bettigniesb, R., Guillerezb, S., \& Defranouxc, C. (2007). Modeling the short-circuit current density of polymer solar cells based on P3HT:PCBM blend. Solar Energy Materials and Solar Cells, Vol. 91, No. 5, (6 March 2007), pp. (405-410), ISSN 0927-0248

Mühlschlegel, P., Eisler, H.-J., Martin, O. J. F., Hecht, B., \& Pohl, D. W. (2005). Resonant Optical Antennas. Science, Vol. 308, No. 5728, (10 June 2005), pp. (1607-1609), ISSN 0036-8075

Noginov, M. A., Zhu, G., Belgrave, A.M., Bakker, R., Shalaev, V. M., Narimanov, E. E., Stout, S., Herz, E., Suteewong, T., \& Wiesner U. (2009). Demonstration of a spaser-based nanolaser. Nature, Vol. 460, (27 August 2009), pp. (1110-1112), ISSN 0028-0836

Nollé, É. L., Shchelev, M. Ya. (2004). Photoelectron emission caused by surface plasmons in silver nanoparticles. (2004). Technical Physics Letters, Vol. 30, No.4, (April 2004), pp.( 304-306), ISSN 1063-7850

Nollé, É. L., Shchelev, M. Ya. (2005). Photoelectron emission from granulated gold films activated by cesium and oxygen. Technical Physics, Vol. 50, No. 11, (November 2005), pp.(1528-1530), ISSN 1063-7842

Nollé, É. L. (2007). Tunneling photoeffect mechanism in metallic nanoparticles activated by cesium and oxygen. Physics-Uspekhi, Vol. 50, No. 10, (October 2007), pp.(1079-1083), ISSN 0036-8075

Novotny, L., Hecht, B. (2006). Principles of Nano-optics, Cambridge University Press, ISBN 978-052-1832-24-3, Cambridge, UK

Oulton, R. F., Sorger, V. J., Zentgraf, T., Ma, R-M., Gladden, C., Dai, L., Bartal, G., \& Zhang, X. (2009). Plasmon lasers at deep subwavelength scale. Nature, Vol. 461, (1 October 2009), pp. (629-632), ISSN 0028-0836

Piotrowski, J., Galus, W., \& Grudzi, M. (1990). Near room-temperature IR photo-detectors. Infrared Phys.. Vol. 31, No. 1, (January 1990), pp. (1-48), ISSN 1350-4495 
Pors, A., Uskov, A.V., Willatzen, M., \& Protsenko, I. E. (2011). Control of the input efficiency of photons into solar cells with plasmonic nanoparticles. Optics communications, Vol. 284, No. 8, (15 April 2011), pp. (2226-2229), ISSN 0030-4018

Protsenko, I. E., Uskov, A. V., Zaimidoroga, O. A., Samoilov, V. N., \& O'Reilly E. P. (2005). Dipole nanolaser. Physical Review A, Vol. 71, No. 6, (17 June 2005), pp. (063812 [7 pages]), ISSN 1050-2947

Rand B.P., Peumans, P. \& Forrest, S.R. (2004). Long-range absorption enhancement in organic tandem thin-film solar cells containing silver nanoclusters. Journal of Applied Physics, Vol. 96, No.12, (15 December 2003), pp. (7519-7526), ISSN 0021-8979

Schelev, M. Ya. (2000) Femtosecond photoelectronics - past, present, and future. PhysicsUspekhi, Vol. 43, No.9, (September 2000), pp.(931-946), ISSN 0036-8075

Schuller, J., Barnard, E., Cai, W., Jun, Y., White J., \& Brongersma M. (2010). Plasmonics for extreme light concentration and manipulation. Nature Materials, Vol. 9, No.3, (March 2010), pp. (193-204), ISSN 1476-1122

Soole, J. B. D., Schumacher, H. (1991). InGaAs metal-semiconductor-metal photodetectors for long wavelength optical communications. IEEE Journal of Quantum Electronics, Vol. 27, No.3, (March 1991), pp. (737 - 752), ISSN 0018-9197

Sze S.M., (1981). Physics of semiconductor devices, Wiley, ISBN 978-047-1056-61-4, New York, Chichester, Brisbane, Toronto, Singapore

Wang, F., Ron Shen, Y. (2006). General properties of local plasmons in metal nanostructures. Physical Review Letters, Vol. 97, No. 20, (November 2006), pp. (206806 [4 pages]), ISSN 0031-9007

Weber, M. J. (2002). Handbook of optical materials, CRC Press, Boca Raton, London, New York, Washington, D.C., ISBN 978-084-9335-12-9

Westphalen, M., Kreibig, U., Rostalski, J., Lüth, H., \& Meissner, D. (2000). Metal cluster enhanced organic solar cells. Sol.Energy Mater. Sol. Cells, Vol. 61, No.1, (15 February 2000), pp. (97-105), ISSN 0021-8979

Yu, Z., Veronis, G., Fan, S., \& Brongersma, M. (2006). Design of midinfrared photodetectors enhanced by surface plasmons on grating structures. Applied Physics Letters, Vol. 89, No.15, (9 October 2006), pp. (151116 3 pages), ISSN 0003-6951 


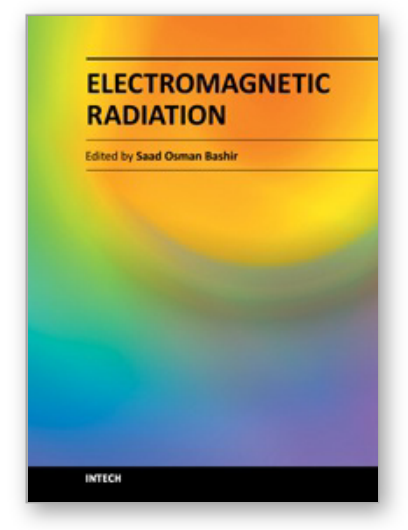

\author{
Electromagnetic Radiation \\ Edited by Prof. S. O. Bashir
}

ISBN 978-953-51-0639-5

Hard cover, 288 pages

Publisher InTech

Published online 05, June, 2012

Published in print edition June, 2012

The application of electromagnetic radiation in modern life is one of the most developing technologies. In this timely book, the authors comprehensively treat two integrated aspects of electromagnetic radiation, theory and application. It covers a wide scope of practical topics, including medical treatment, telecommunication systems, and radiation effects. The book sections have clear presentation, some state of the art examples, which makes this book an indispensable reference book for electromagnetic radiation applications.

\title{
How to reference
}

In order to correctly reference this scholarly work, feel free to copy and paste the following:

Igor Protsenko and Alexander Uskov (2012). Photoemission from Metal Nanoparticles, Electromagnetic Radiation, Prof. S. O. Bashir (Ed.), ISBN: 978-953-51-0639-5, InTech, Available from:

http://www.intechopen.com/books/electromagnetic-radiation/photoemission-from-metal-nanoparticles

\section{INTECH}

open science | open minds

\author{
InTech Europe \\ University Campus STeP Ri \\ Slavka Krautzeka 83/A \\ 51000 Rijeka, Croatia \\ Phone: +385 (51) 770447 \\ Fax: +385 (51) 686166 \\ www.intechopen.com
}

\author{
InTech China \\ Unit 405, Office Block, Hotel Equatorial Shanghai \\ No.65, Yan An Road (West), Shanghai, 200040, China \\ 中国上海市延安西路65号上海国际贵都大饭店办公楼 405 单元 \\ Phone: +86-21-62489820 \\ Fax: +86-21-62489821
}


(C) 2012 The Author(s). Licensee IntechOpen. This is an open access article distributed under the terms of the Creative Commons Attribution 3.0 License, which permits unrestricted use, distribution, and reproduction in any medium, provided the original work is properly cited. 